\title{
Advancing Alzheimer's disease diagnosis, treatment, and care: Recommendations from the Ware Invitational Summit
}

Mary D. Naylor ${ }^{\mathrm{a}, \mathrm{b}, \mathrm{c}, *}$, Jason H. Karlawish ${ }^{\mathrm{a}, \mathrm{d}, \mathrm{e}}$, Steven E. Arnold ${ }^{\mathrm{a}, \mathrm{f}, \mathrm{g}}$, Ara S. Khachaturian ${ }^{\mathrm{h}}$, Zaven S. Khachaturian ${ }^{h}$, Virginia M.-Y. Lee ${ }^{\mathrm{a}, \mathrm{i}, \mathrm{j}}$, Matthew Baumgart ${ }^{\mathrm{k}}$, Sube Banerjee , $^{\mathrm{l}}$ Cornelia Beck ${ }^{\mathrm{m}}$, Kaj Blennow ${ }^{\mathrm{n}}$, Ron Brookmeyer ${ }^{\mathrm{o}}$, Kurt R. Brunden ${ }^{\mathrm{a}, \mathrm{i}, \mathrm{j}}$, Kathleen C. Buckwalter ${ }^{\mathrm{p}}$, Meryl Comer ${ }^{\mathrm{q}}$, Kenneth Covinsky ${ }^{\mathrm{r}}$, Lynn Friss Feinberg ${ }^{\mathrm{s}}$, Giovanni Frisoni ${ }^{\mathrm{t}}$, Colin Green ${ }^{\mathrm{u}}$, Renato Maia Guimaraes ${ }^{v}$, Lisa P. Gwyther ${ }^{w}$, Franz F. Hefti ${ }^{\mathrm{x}}$, Michael Hutton ${ }^{\mathrm{y}}$, Claudia Kawas ${ }^{\mathrm{z}}$, David M. Kent ${ }^{\mathrm{aa}}$, Lewis Kuller ${ }^{\mathrm{bb}}$, Kenneth M. Langa ${ }^{\mathrm{cc}}$, Robert W. Mahley ${ }^{\mathrm{dd}}$, Katie Maslow ${ }^{\mathrm{ee}}$, Colin L. Masters ${ }^{\mathrm{ff}}$, Diane E. Meier ${ }^{\mathrm{gg}}$, Peter J. Neumann ${ }^{\text {aa }}$, Steven M. Paul ${ }^{\text {hh }}$, Ronald C. Petersen ${ }^{\text {ii }}$, Mark A. Sager ${ }^{\mathrm{jj}}$, Mary Sano ${ }^{\mathrm{gg}}$, Dale Schenk ${ }^{\mathrm{kk}}$, Holly Soares ${ }^{\mathrm{ll}}$, Reisa A. Sperling ${ }^{\mathrm{mm}}$, Sidney M. Stahl ${ }^{\mathrm{nn}}$, Vivianna van Deerlin ${ }^{\mathrm{a}, \mathrm{i}, \mathrm{j}}$, Yaakov Stern ${ }^{\mathrm{oo}}$, David Weir ${ }^{\mathrm{pp}}$, David A. Wolk ${ }^{\mathrm{g}}$, John Q. Trojanowski ${ }^{\mathrm{a}, \mathrm{i}, \mathrm{j}}$

\footnotetext{
${ }^{a}$ Institute on Aging, University of Pennsylvania, Philadelphia, PA, USA

${ }^{b}$ School of Nursing, University of Pennsylvania, Philadelphia, PA, USA

${ }^{c}$ NewCourtland Center for Transitions and Health, University of Pennsylvania School of Nursing, Philadelphia, PA, USA

${ }^{d}$ Department of Medical Ethics and Health Policy, Perelman School of Medicine, University of Pennsylvania, Philadelphia, PA, USA ${ }^{e}$ Department of Medicine, Division of Geriatric Medicine, Perelman School of Medicine, University of Pennsylvania, Philadelphia, PA, USA

${ }^{f}$ Department of Psychiatry Section of Geriatric Psychiatry, Perelman School of Medicine, University of Pennsylvania, Philadelphia, PA, USA

${ }^{g}$ Department of Neurology, Perelman School of Medicine, University of Pennsylvania, Philadelphia, PA, USA

${ }^{h}$ Prevent Alzheimer's Disease by 2020 (PAD 2020), Rockville, MD

${ }^{i}$ Department of Pathology and Laboratory Medicine, Perelman School of Medicine, University of Pennsylvania, Philadelphia, PA, USA

${ }^{j}$ Center for Neurodegenerative Disease Research, Perelman School of Medicine, University of Pennsylvania, Philadelphia, PA, USA

${ }^{k}$ Alzheimer's Association, Chicago, IL, USA

lInstitute of Psychiatry, King's College London, London, UK

${ }^{m}$ University of Arkansas for Medical Sciences, Little Rock, AR, USA

${ }^{n}$ The Sahlgrenska Academy, University of Göteborg, Göteborg, Sweden

${ }^{o}$ School of Public Health, University of California at Los Angeles, Los Angeles, CA, USA

${ }^{p}$ University of Iowa, Iowa City, IA, USA

${ }^{q}$ Geoffrey Beene Foundation Alzheimer's Initiative, New York, NY, USA

${ }^{r}$ University of California at San Francisco, San Francisco, CA, USA

${ }^{s}$ AARP Public Policy Institute, Washington DC, USA

${ }^{t}$ The National Centre for Alzheimer's and Mental Diseases, IRCCS Fatebenefratelli, Brescia, Italy

"Peninsula Medical School, University of Exeter, Exeter, UK

${ }^{v}$ International Association of Gerontology and Geriatrics, Brasilia, Brazil

${ }^{w}$ Duke University Aging Center, Durham, NC, USA

${ }^{x}$ Acumen Pharmaceuticals, Inc., Livermore, CA, USA

${ }^{y}$ Eli Lilly, Inc., Windlesham, Surrey, UK

${ }^{z}$ University of California, Irvine, Irvine, CA, USA

${ }^{a}$ Tufts Medical Center, Boston, MA, USA

${ }^{b b}$ School of Public Health, University of Pittsburgh, Pittsburgh, PA, USA

${ }^{c c}$ University of Michigan and Veteran's Affairs Ann Arbor Health Care System, Ann Arbor, MI, USA

${ }^{d d}$ J. David Gladstone Institutes, San Francisco, CA, USA

${ }^{e e}$ Institute of Medicine, Washington DC, USA

${ }^{f f}$ Mental Health Research Institute, University of Melbourne, Melbourne, Australia
}

*Corresponding author. Tel.: 215-898-6088; Fax: 215-573-4225.

E-mail address: naylor@nursing.upenn.edu 


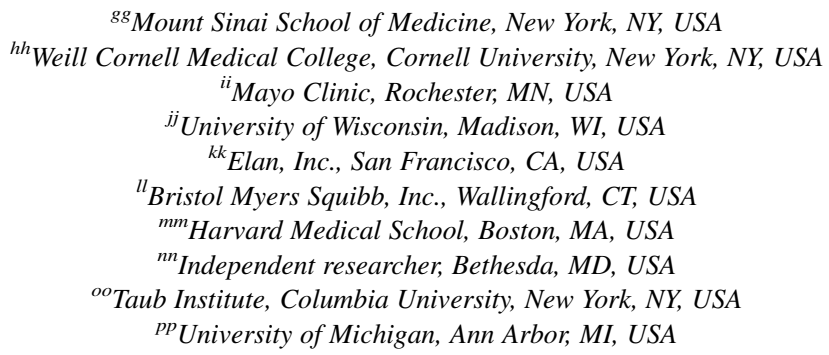

Abstract

To address the pending public health crisis due to Alzheimer's disease (AD) and related neurodegenerative disorders, the Marian S. Ware Alzheimer Program at the University of Pennsylvania held a meeting entitled "State of the Science Conference on the Advancement of Alzheimer's Diagnosis, Treatment and Care," on June 21-22, 2012. The meeting comprised four workgroups focusing on Biomarkers; Clinical Care and Health Services Research; Drug Development; and Health Economics, Policy, and Ethics. The workgroups shared, discussed, and compiled an integrated set of priorities, recommendations, and action plans, which are presented in this article.

(C) 2012 The Alzheimer's Association. All rights reserved.

\section{Alzheimer's disease-A growing crisis}

The aging of populations globally has precipitated a demographic "sea change" that is having a profound impact on societies worldwide and is the most powerful driver of the increase in age-related disorders, including Alzheimer's disease (AD) and related neurodegenerative disorders. Addressing these changes is among the most important challenges facing scientists, health-care providers, policymakers, the business community, and governments worldwide [1-3].

To address the pending public health crisis due to $\mathrm{AD}$ and related neurodegenerative disorders, the Marian S. Ware Alzheimer Program at the University of Pennsylvania held a meeting entitled "State of the Science Conference on the Advancement of Alzheimer's Diagnosis, Treatment and Care," on June 21-22, 2012. The meeting comprised four workgroups focusing on Biomarkers; Clinical Care and Health Services Research; Drug Development; and Health Economics, Policy, and Ethics-all of which must be addressed simultaneously. The intent of the Ware Summit was to develop both cross-cutting and domain-specific recommendations for a range of stakeholders, including the scientific community, policymakers, legislators, advocacy groups, and clinicians. Toward this end, each workgroup met via conference call over several months to develop a preliminary set of recommendations (Appendix 1). At the conference, these recommendations were shared, discussed, and compiled into an integrated set of priorities, recommendations, and action plans, which are presented in this work.

While the four workgroups each developed focused recommendations reflecting their individual perspectives (Appendix 2), they reached remarkable consensus on the core principles and priorities that must be addressed both short and long term. Attention to these issues would enable us to provide good quality care for affected patients and families, advance our understanding of the pathophysiology and natural history of $\mathrm{AD}$ and other dementias, develop effective treatments to slow or prevent these diseases, and translate scientific advances successfully into policy and practice. The overarching goals identified at the Ware Summit (Table 1) mirror those reflected in the United States National Plan to address Alzheimer's Disease (USNPAAD, or the "National Plan") [1], released May 15, 2012 by the U.S. Department of Health and Human Services (DHHS) as part of the National Alzheimer's Project Act (NAPA). The National Plan outlines a comprehensive approach of prevention and treatment for AD by 2025 .

Recognizing the urgency of the challenges faced by the Alzheimer's field as well as the population at large, the recommendations outlined in this study call for a number of immediate policy changes as well as introduction of legislative initiatives that would produce effective and sustainable longterm changes in efforts to address the oncoming public health challenges. These recommendations should provide a catalyst to drive research planning, develop alternative care-delivery models, and allocate resources to achieve these goals.

\section{Core principles}

Underlying these recommendations are several core principles intended to advance the clinical delivery of care and scientific understanding of $\mathrm{AD}$ and related disorders. Efforts to link science with care and engaging the participation of patients, families, scientists, pharmaceutical companies, regulatory agencies, and advocacy organizations were seen by conference participants as path-breaking and 
Table 1

Overall goals identified at the Ware Invitational Summit

1. Identify and develop novel treatments, including non-pharmacologic treatments for AD within the next decade, while continuing to focus on ameliorating or preventing $\mathrm{AD}$.

2. Improve the efficiency and knowledge gained from clinical trials.

3. Enable better patient options through earlier identification of individuals at risk for AD or those in whom the neurodegenerative process has already begun

4. Provide high-quality, cost-effective, and ethical care to persons with dementia and their family members across the trajectory of the illness.

instrumental in developing better outcomes for people with $\mathrm{AD}$ as has been demonstrated for cardiovascular disease, diabetes, and cancer. The Alzheimer's field should learn from and expand on this model, keeping in mind the need to:

- Integrate, but not duplicate, services, resources, and research, including building on existing infrastructure where appropriate (e.g., resources available across institutes at the National Institutes of Health [NIH], Veteran's Administration, Center for Medicare and Medicaid Services [CMS], Centers for Disease Control [CDC], Food and Drug Administration [FDA], and the Department of Defense [DoD]).

- Shift resources and care delivery to the community and home, providing "one-stop shopping" that incorporates both medical and social interventions along with research and training to improve the lives of people with $\mathrm{AD}$ and their families.

- Increase awareness among the public, health-care providers, and policymakers.

- Deliver cost-effective diagnostics, treatments, and services.

- Engage patients, family caregivers, and advocacy groups in the decision-making process.

- Focus on affected persons and their families across the trajectory of AD disease. This requires a better understanding of the natural history of the disease and effects of multimorbidity.

- Focus on drug development and biomarker research with an aim toward reducing the incidence and progression of dementia.

- Ensure that addressing dementia is a priority across all segments of societies and governments both nationally and internationally.

\section{Recommendations}

The complete list of recommendations made by the four individual workgroups is given in Appendix 2. These have been reorganized and integrated to address the goals outlined in Table 1. Recommendations across all workgroups and goals are compiled in Table 2, with the highest priority recommendations noted. In making these recommendations, conference participants considered both the urgency of the public health challenges facing the nation and world as well as the reality of shrinking federal budgets. Yet, despite the challenging funding environment, there is an undeniable need for increased investment for
Alzheimer's research, including basic, translational, clinical, and health-services research, and attention to caregivers.

\subsection{Identifying and developing novel treatments that ameliorate or prevent $A D$ within the next decade}

The effort to develop disease-modifying therapies for $\mathrm{AD}$ has reached a crisis [2] and finding a path forward to overcome this impasse will require the collaborative efforts of the research, clinical, pharmaceutical, and regulatory communities, as well as policymakers, to identify barriers and solutions at each stage of drug development. Moreover, there has been a shift in thinking about the treatment of AD, away from the possibility that a single drug could ameliorate the disease. Today, there is increasing recognition of the complexity of the disease, the likelihood that multiple treatments will be needed at different phases of the disease, and that treatment will require more than drugs. Toward this end, one of the highest priorities is to better understand the pathophysiology of $\mathrm{AD}$ and related disorders by broadening the research focus to encompass cardiovascular, metabolic, and inflammatory diseases, all of which play important roles in the development of dementia.

Biomarkers have become essential tools in drug development, both for enriching study populations with subjects likely to respond to the drug and for monitoring response to treatment. Objectively monitoring changes in $\mathrm{AD}$ is extremely valuable, but may not be sufficient for monitoring progression of disease pathology or neurodegeneration per se. Biomarkers that separate $\mathrm{AD}$ and $\mathrm{AD}$ dementia from other aging-related dementias would also be valuable.

In contrast to early-onset familial $\mathrm{AD}$, the far more common and sporadic late-onset $\mathrm{AD}$ (LOAD) is a multifactorial disease intermixed with universal senescent processes. Frequently, other common pathologic conditions, such as vascular disease or other proteinopathies due to $\alpha$-synuclein or TAR DNA protein binding 43 (TDP-43), which are characteristic of Parkinson's disease (PD) and frontotemporal degeneration (FTD), respectively, coexist with $\mathrm{AD}$ in the same patient. At present, we do not have biomarkers to differentiate these conditions, quantify the relative contributions of different disease processes, or measure how active any one might be relative to another. In addition, new biomarkers that assess other aspects of the disease, such as synaptic function and inflammation, are needed to improve prediction beyond what is available now, to determine contributions from other diseases, and to better assess disease 
Table 2

Goals and recommendations

\begin{tabular}{lcc}
\hline Goal & Priority recommendations & Further recommendations \\
\hline $\begin{array}{c}\text { Identify and develop novel treatments, including } \\
\text { non-pharmacologic treatments for AD within } \\
\text { the next decade while continuing to focus on }\end{array}$ & $\begin{array}{c}\text { Increase NIH grant support for research on } \\
\text { understanding AD disease mechanisms and } \\
\text { ameliorating or preventing AD. }\end{array}$ & $\begin{array}{c}\text { Develop new biomarkers, especially those that } \\
\text { reflect current disease activity (synaptic integrity, }\end{array}$ \\
& $\begin{array}{l}\text { least } \$ 50 \mathrm{M} / \text { year. } \\
\text { Effect revisions of the regulatory process in order to } \\
\text { speed the translation of research discoveries into }\end{array}$ & $\begin{array}{c}\text { Develop better cognitive and functional markers of } \\
\text { the early stages. }\end{array}$
\end{tabular}

speed the translation of research discoveries into clinical practice and incentivize industry to develop AD drugs, including consideration of alternative clinical endpoints.

Establish the relationship between biomarkers and cognitive and functional markers.

Improve the efficiency and knowledge gained from clinical trials.

Complete standardization and validation of existing biomarkers of interest.

Incorporate $\mathrm{AD}$ biomarker assessment into all $\mathrm{AD}$ clinical trials, including those targeting early presymptomatic stages.

Expand longitudinal studies that use standardized protocols and include multiple biomarkers to include subjects that better reflect the general population.

Enable better patient care through earlier identification of individuals at risk for $\mathrm{AD}$ or those in whom the neurodegenerative process has already begun.

Establish a national/international population-based registry of older adults with and without dementia, including geographically and culturally different populations and individuals with comorbid conditions.

Develop a risk stratification model that incorporates demographic, genetic, biologic, cognitive, and environmental markers.

Urge all clinical trials involving older patients to include cognitive markers and $\mathrm{AD}$ biomarkers as part of their assessments.

Require data from clinical trials of both drugs and biomarkers, including Phase IV data, to be placed in a shared database to ensure that lessons learned are shared within the $\mathrm{AD}$ research community.

Conduct longitudinal population-based studies to clarify the natural history of $\mathrm{AD}$ and other dementias using clinical measures, cognitive tests, patient reported outcomes, and biomarkers.

Establish a detection and treatment regimen for preclinical AD individuals.

Develop best practice standards on when to order biomarker test, what and how to disclose and interpret results.

Develop a training and certification program for conducting biomarker tests, interpreting data, communicating biomarker data, and quality assurance.

Provide high-quality, cost-effective, and ethical care to persons with dementia and their family

Increase NIH and other sources of support to advance person- and population-centered, highmembers across the trajectory of the illness. value care for patients afflicted with $\mathrm{AD}$ and their family caregivers.

Conduct health services research on diverse delivery models, including a full assessment of existing best practices, such as the Program of All-Inclusive Care for the Elderly (PACE), palliative care, and international models.

Review and propose appropriate revisions to existing laws, institutions, and social structures-such as employers, insurers, housing, and schools- to assure that they will protect the rights and interests of persons who have had an AD biomarker test or other test that places them at high risk of developing $\mathrm{AD}$.

Package best practices into technical assistance skill sets for families and clinicians.

Consider legislation requiring that, until the clinical value of biomarkers is fully understood, information gained through biomarker studies cannot be considered in insurance or employment decisions.

Promote legislation that accelerates implementation of proven effective and efficient care models for people with dementia and their families.

drug pipeline has resulted in a dramatically weakened indus-

progression and treatment efficacy. Biomarkers that indicate the degree of disease or neurodegeneration at any given point after diagnosis could help guide treatment and assess treatment effectiveness in clinical trials.

The current situation for the pharmaceutical industry to bring a new medical entity (NME) to market is both expensive and extremely long, with the average time now estimated to be 10 to 12 years and the cost about $\$ 1.8$ billion and rising rapidly [2]. More importantly, however, a series of patent expirations on blockbuster drugs and a dwindling try, which could also adversely affect public health efforts to control AD. Drug discovery may need to transition to a new model where discovery and early development of welldefined therapeutic products emanates from academic or nonprofit institutions, or small biotechnology companies [3]. In this framework, federal money will help leverage and launch new public-private partnerships, similar to the AD Neuroimaging Initiative (ADNI) [4], and could also play important roles in filling the precompetitive space [5]. 
Recommendations aimed at finding novel treatments to ameliorate $\mathrm{AD}$ within the next decade include:

- Increasing NIH grant support for research on understanding $\mathrm{AD}$ disease mechanisms and identifying new AD drug discovery targets by $\geq \$ 50$ million/year.

- Developing new biomarkers, especially those that reflect current disease activity (synaptic integrity, TDP-43, vascular disease, $\alpha$-synuclein).

- Developing better cognitive and functional markers sensitive to change in early stages.

- Establishing the relationship between biomarkers and cognitive and functional markers.

- Exploring revisions of the regulatory process to speed the translation of research discoveries into clinical practice and incentivize pharmaceutical companies to develop AD drugs.

\subsection{Improving the efficiency and knowledge gained from clinical trials}

Recent disappointing clinical trials of AD pharmacologic treatments have slowed new investment into AD drug development. Regardless of whether drug trials currently underway demonstrate efficacy, there is a growing consensus within the AD clinical research community that clinical trials must be redesigned for greater efficiency and that the data gathered in these trials must be carefully analyzed to provide lessons for future drug development. It is essential to improve research and development (R\&D) productivity by increasing the efficiency of work in process and the probability of success, and by decreasing Phase II and III attrition [2], or by changing the current regulatory paradigm. For example, neurodegenerative disease biomarkers provide new opportunities to shorten the length of clinical trials so that they do not consume the period of exclusivity on a new drug. Concerns regarding the length of clinical trials have been compounded by patent law changes that will take effect in March 2013 [2], which may shorten effective patent life after the lengthy product approval process.

Public-private partnerships, such as ADNI, have proven to be effective models for collecting and sharing data in precompetitive space. ADNI and its international partners (Worldwide ADNI), as well as the Alzheimer's Biomarker Standardization Initiative (ABSI) [6] and the Alzheimer's Association's Global QC program for AD CSF biomarkers [7], have also led the way in standardization and validation of biomarkers, and this process should be completed. International standardization of AD biomarkers could impact the field in much the same way that standardization of cholesterol or hemoglobin A1c measurement led to widespread use of these tests in clinical trials and the discovery and development of effective treatments for cardiovascular disease and diabetes.

Because cognition is currently thought to be the most clinically relevant characteristic of the disease, it is also im- perative that more sensitive and specific cognitive and functional markers be identified, standardized, validated, and correlated with other biomarkers across the continuum of the disease process, and that these cognitive markers are also incorporated into all clinical trials. Moreover, data collected in these trials can prove extremely valuable in planning future drug discovery efforts, and should be made available to other researchers, with appropriate compensation for sponsors (e.g., prompt release of clinical data from placebo-treatment groups). Recommendations aimed at improving the efficiency of and knowledge gained from clinical trials include:

- Completing standardization and validation of existing biomarkers of interest.

- Incorporating AD biomarker assessment into all clinical trials of disease-modifying and symptomatic AD drugs.

- Urging all trials involving older patients to incorporate cognitive and functional markers and AD biomarkers as part of their assessments.

- Expanding longitudinal studies that use standardized protocols and include multiple biomarkers to include subjects that better reflect the general population.

- Requiring data from clinical trials, including Phase IV data, to be placed in a shared database to ensure that lessons learned are shared within the $\mathrm{AD}$ research community without impinging on the intellectual property rights of sponsors.

\subsection{Enabling better patient management through early identification}

There is a growing consensus in the AD clinical research community of the need to identify individuals at risk for $\mathrm{AD}$ or those in whom the neurodegenerative process has already begun [8-11]. New diagnostic criteria proposed by three workgroups convened by the National Institute on Aging in partnership with the Alzheimer's Association (NIA-AA) [12-15], as well as by an International Working Group (IWG) [16], recognized the value of incorporating biomarkers of $\mathrm{AD}$ and neurodegeneration in research studies, but concluded that they have not been sufficiently standardized or validated for use in clinical care. The field is rapidly moving forward, with the goal of using biomarkers to ascertain $\mathrm{AD}$ as a diagnosis of inclusion with a level of certainty, rather than a diagnosis to be made after excluding other disorders. However, the consensus at this time is that the science does not support the widespread clinical application of a biomarker-based diagnosis.

The NIA-AA committees distinguished three stages of disease: (1) a preclinical phase, where the pathophysiologic processes of $\mathrm{AD}$ have begun before any evident signs of cognitive impairment; (2) a prodromal or $M C I$ due to $A D$ phase, where there is evident decline in memory or other cognitive functions; and (3) $A D$ dementia, where cognitive and 
functional deterioration have reached a threshold at which an individual needs help with activities of daily living.

Although substantial data indicate that leading cerebrospinal fluid (CSF) and neuroimaging biomarkers provide clinically useful information for patients with evident cognitive impairment (i.e., mild cognitive impairment and dementia), current longitudinal data are not sufficient to warrant the use of AD biomarkers in asymptomatic individuals [15]. About one third of older adults with normal cognition exhibit abnormal CSF $A \beta$ levels or $A \beta$ on positron emission tomography (PET) scans; however, it is not yet known whether abnormal AD biomarkers in asymptomatic people indicate a disease process that will manifest as mild cognitive impairment (MCI) and $\mathrm{AD}$ at some future point and, if so, when. Although ADNI has been extraordinarily successful in identifying, standardizing, and validating biomarkers, the population studied does not necessarily reflect the general population. Thus, to establish appropriate biomarker cutoffs for both clinical trials and clinical usage, it is imperative to conduct longitudinal, population-based studies with standardized protocols and multiple biomarkers and cognitive markers. Establishing an international population-based registry of older adults, including geographically and culturally different populations and individuals with comorbid conditions, is thus a high-priority recommendation from this conference [17]. This registry could take advantage of existing clinical databases such as those managed by the Department of Veteran's Affairs and the Center for Medicare and Medicaid Services, and could be modeled after other large longitudinal studies such as the Framingham Study, the Cardiovascular Health Study, the Health and Retirement Study, and other population-based studies of aging (summarized in Ref. [18]).

Using biomarkers to define risk, however, presents several concerns, particularly because a therapy that impacts an $\mathrm{AD}$ biomarker is not certain to translate into clinically meaningful endpoints [19]. The experience of other biomarker-based processes demonstrates the need for caution in disseminating a biomarker into clinical practice without clarifying its value [20]. Clinicians do not yet have a good way to predict the future of $\mathrm{AD}$, including the speed of progression and the extent that function will be affected.

If an effective disease-modifying therapy is reported, research needs to address how it will change AD. In particular, as ongoing and planned research enhances our understanding of the preclinical stages of $\mathrm{AD}$, both how we think about the disease and its treatment will change. What was once a disease defined by clinical signs and symptoms, with treatments designed to reduce these clinical features, will become a disease defined by the risk of developing these signs and symptoms, and once they occur, their risk of worsening. The concept of successful treatment (including nonpharmacologic treatment) will then be transformed to mean reducing risk and improving quality of life, and it will be imperative that the prevention of signs and symptoms is shown to be both clinically meaningful and cost effective.
As the value of biomarkers and other risk factors emerges and becomes clinically useful, patients and society will be better served if we think of biomarkers not as labels of a category, such as "preclinical AD," but rather as one, yet not the only, measure of risk for disability as a result of progressive cognitive decline. To make the best use of established and emerging biomarkers and other risk factors for $\mathrm{AD}$, a major goal of the $\mathrm{AD}$ research community should be to develop and validate a risk-stratification model for the development of endpoints, such as MCI or dementia, to guide 1) clinical and care management decisions by patients, families, and health-care providers; 2) policymaking; and 3) research to test new interventions. Such a risk-prediction model, which incorporates drugs, clinical trials, and biomarkers [21], holds substantial promise for improving public health. Moreover, it is possible that certain biomarkers will ultimately identify individuals who will progress to $\mathrm{AD}$ and hence prove critically important for identifying early treatment and intervention strategies as these emerge, akin to how a Pap smear is currently used to identify women at risk of cervical cancer. The Economics, Ethics and Health Policy Workgroup drew on their collective expertise in both neurodegenerative diseases and other diseases of aging, such as cardiovascular disease, osteoporosis, and cancer, to propose how the promise of this risk-based model can be realized.

The risk-stratification model should be developed using data from representative population-based samples so as to accurately represent the clinical complexity of typical older adults, and the resulting competing health risks (e.g., heart disease, diabetes, cancer) that may have a major impact on life expectancy and quality of life, and therefore, the efficacy and value of drug and other interventions to prevent or treat AD. Further, the risk-stratification models should be in the public domain, and should be evaluated and updated by a public body (e.g., DHHS) using established standards for the reliable and valid measurement of $\mathrm{AD}$ risk factors, including the standardization of AD biomarkers. The ongoing evaluation of risk-stratification models should include assessments of the costs (e.g., from unnecessary treatment and treatment side-effects) that result from misclassification when using the models. Recommendations aimed at enabling better patient care through early identification include:

- Establishing a national/international population-based registry of older adults, including geographically and culturally different populations and individuals with comorbid conditions.

- Conducting longitudinal population-based studies to clarify the natural history of $\mathrm{AD}$ and other dementias using clinical measures, cognitive tests, and biomarkers.

- Developing best practice standards on when to order the biomarker test, and what and how to disclose and interpret results. 
- Developing a training and certification program for conducting biomarker tests, interpreting data, communicating biomarker data, and quality assurance.

- Developing a risk-stratification model that incorporates demographic, genetic, biologic, cognitive, and environmental risk factors.

\subsection{Providing quality, cost-effective, and ethical care to persons with dementia and their family members}

$\mathrm{AD}$ and other dementias represent the most urgent problem facing the aging population, but these health challenges exist within the larger context of aging, multiple morbidity and resulting disabilities, and social risks. Providing good quality and cost-effective care to persons with dementia and their families means providing care across the continuum of the illness, beginning at the "something-is-wrong" stage. This will require accelerating advances in knowledge and policy that would foster care-delivery systems more closely aligned with people's needs throughout their health and illness trajectories, a better understanding of current best practices, and developing and implementing new strategies to build and expand the workforce capacity to create and support a high-value care system for people with $\mathrm{AD}$ and their family caregivers.

Cognitive impairment in combination with other acute and chronic illnesses complicates the care of patients, although the precise effect of coexisting chronic conditions on health-care utilization and delivery is not well understood [22]. A truly responsive health-care system needs to address the needs of family members and other caregivers along an individualized and changing illness trajectory and in multiple contexts. As people move along this continuum, their needs become more complex often because many chronic problems are progressive in nature. In addition, limited social support, health literacy and equity, language and cultural barriers, financial limitations, and other factors may exacerbate the nature and intensity of these needs.

Bringing care to persons with dementia and their families can best be realized by integrating in one place health-care and social services as well as research and training programs. Numerous models for such community-based health-care delivery exist internationally, including, in the United States, the Program of All-Inclusive Care for the Elderly (PACE), and various palliative-care programs. The best of these programs join proactively with community partners to enable preservation of cognitive and physical health through primary prevention strategies such as public health awareness programs and fitness training [23].

To identify the model that best fits the needs of patients with dementia and their families, health services research is needed to explore diverse delivery models and their applicability at different stages of illness and with different levels of family need and capability. These care models then need to be tested with regard to their effects on key clinical and utilization metrics within the overall population and among different subgroups. Further, there is a need to better understand the goals identified as most meaningful by the people served - an enhanced care experience, improved health and quality of life, and/or survival-while choosing wisely with finite resources [17].

Following the identification of best practices, there will be a need to integrate these priorities into the DHHS/ NAPA National Plan and articulate the value proposition to key stakeholders, including governmental organizations, legislators, advocacy groups, and the public at large. Technical assistance skill sets designed to meet the needs of patients, families, and clinicians can be developed to speed implementation of best practices into the community.

Optimal care of patients and families also requires protection of their rights and interests, attention to issues, such as stigma, and protection against possible misuse of clinical information, including results from biomarker tests and risk assessment. Thus, we recommend review and possible revision of existing laws that pertain to institutions and social structures (such as insurance and employment) to ensure protection of patients and their families. Recommendations to ensure quality, cost-effective care, and ethical care to persons with dementia and their family members include:

- Conducting health services research on diverse delivery models, including a full assessment and dissemination of evidence-based current best practices.

- In order to protect the rights and interests of patients who have had biomarker testing and to prevent misuse of biomarker data, thorough evaluation and appropriate revisions are needed of existing laws, institutions, and social structures.

- Legislation should be considered immediately that, until the clinical value of biomarkers is fully understood, information gained through biomarker studies cannot be considered in insurance or employment decisions.

\section{Conclusions}

The goal of the Ware Invitational Summit on the Advancement of Alzheimer's Diagnosis, Treatment, and Care was to formulate a set of recommendations that will inform efforts around the world to address the escalating $\mathrm{AD}$ public health crisis. This study represents the work of a multidisciplinary group of researchers, advocates, and clinicians from academia, industry, and advocates. These experts first addressed concerns specific to four different domains: Drug Development; Biomarkers; Clinical Care and Health Services Research; and Economics, Ethics, and Policy. This group subsequently came together to incorporate their recommendations into a fully integrated and comprehensive strategy, which is presented here to the field at large as well as to individual stakeholder groups, including the leadership of DHHS/NAPA, the NIH, policymakers, researchers, clinicians, and advocacy and patient groups. 
Implementing many of these recommendations, including increased research funding, will require creative thinking, repurposing of existing funds, vigilance in reducing waste, and a constant focus on cost effectiveness. The enormity and complexity of AD demands no less.

\section{Acknowledgments}

The authors thank Lisa J. Bain for editorial assistance. The authors also thank the Marian S. Ware 2006 CWG Charitable Lead Annuity Trust for sponsoring this program.

\section{References}

[1] United States Department of Health and Human Services, Assistant Secretary for Planning and Evaluation: United States national plan to address Alzheimer's disease, http://aspe.hhs.gov/daltcp/napa/ NatlPlan.pdf, Accessed May 16, 2012. 2012.

[2] Paul SM, Mytelka DS, Dunwiddie CT, Persinger CC, Munos BH, Lindborg SR, Schacht AL. How to improve R\&D productivity: The pharmaceutical industry's grand challenge. Nat Rev Drug Discov 2010;9:203-14.

[3] Cuatrecasas P. Interview with Pedro Cuatrecasas. Nat Rev Drug Dis$\operatorname{cov} 2009 ; 8: 446$.

[4] Weiner MW, Veitch DP, Aisen PS, Beckett LA, Cairns NJ, Green RC, Harvey D, Jack CR, Jagust W, Liu E, et al. The Alzheimer's Disease Neuroimaging Initiative: A review of papers published since its inception. Alzheimers Dement 2012;8:S1-68.

[5] Trojanowski JQ, Arnold SE, Karlawish JH, Brunden K, Cary M, Davatzikos C, Detre J, Gaulton G, Grossman M, Hurtig H, et al. Design of comprehensive Alzheimer's disease centers to address unmet national needs. Alzheimers Dement 2010;6:150-5.

[6] Vanderstichele H, Bibl M, Engelborghs S, Le Bastard N, Lewczuk P, Molinuevo JL, Parnetti L, Perret-Liaudet A, Shaw LM, Teunissen C, et al. Standardization of preanalytical aspects of cerebrospinal fluid biomarker testing for Alzheimer's disease diagnosis: a consensus paper from the Alzheimer's Biomarkers Standardization Initiative. Alzheimers Dement 2012;8:65-73.

[7] Mattsson N, Andreasson U, Persson S, Arai H, Batish SD, Bernardini S, Bocchio-Chiavetto L, Blankenstein MA, Carrillo MC, Chalbot S, et al. The Alzheimer's Association external quality control program for cerebrospinal fluid biomarkers. Alzheimers Dement 2011; 7:386-95.

[8] Khachaturian ZS, Barnes D, Einstein R, Johnson S, Lee V, Roses A, Sager MA, Shankle WR, Snyder PJ, Petersen RC, et al. Developing a national strategy to prevent dementia: Leon Thal Symposium 2009. Alzheimers Dement 2010;6:89-97.

[9] Khachaturian ZS, Khachaturian AS, Thies W. The draft "National Plan" to address Alzheimer's disease-National Alzheimer's Project Act (NAPA). Alzheimers Dement 2012;8:234-6.

[10] Khachaturian ZS, Petersen RC, Snyder PJ, Khachaturian AS, Aisen P, de Leon M, Greenberg BD, Kukull W, Maruff P, Sperling RA, et al.
Developing a global strategy to prevent Alzheimer's disease: Leon Thal Symposium 2010. Alzheimers Dement 2011;7:127-32.

[11] Khachaturian ZS, Snyder PJ, Doody R, Aisen P, Comer M, Dwyer J, Frank RA, Holzapfel A, Khachaturian AS, Korczyn AD, et al. A roadmap for the prevention of dementia II: Leon Thal Symposium 2008. Alzheimers Dement 2009;5:85-92.

[12] Albert MS, DeKosky ST, Dickson D, Dubois B, Feldman HH, Fox NC, Gamst A, Holtzman DM, Jagust WJ, Petersen RC, et al. The diagnosis of mild cognitive impairment due to Alzheimer's disease: recommendations from the National Institute on Aging-Alzheimer's Association workgroups on diagnostic guidelines for Alzheimer's disease. Alzheimers Dement 2011;7:270-9.

[13] Jack CR Jr, Albert MS, Knopman DS, McKhann GM, Sperling RA, Carrillo MC, Thies B, Phelps $\mathrm{CH}$. Introduction to the recommendations from the National Institute on Aging-Alzheimer's Association workgroups on diagnostic guidelines for Alzheimer's disease. Alzheimers Dement 2011;7:257-62.

[14] McKhann GM, Knopman DS, Chertkow H, Hyman BT, Jack CR Jr, Kawas CH, Klunk WE, Koroshetz WJ, Manly JJ, Mayeux R, et al. The diagnosis of dementia due to Alzheimer's disease: Recommendations from the National Institute on Aging-Alzheimer's Association workgroups on diagnostic guidelines for Alzheimer's disease. Alzheimers Dement 2011;7:263-9.

[15] Sperling RA, Aisen PS, Beckett LA, Bennett DA, Craft S, Fagan AM, Iwatsubo T, Jack CR Jr, Kaye J, Montine TJ, et al. Toward defining the preclinical stages of Alzheimer's disease: recommendations from the National Institute on Aging-Alzheimer's Association workgroups on diagnostic guidelines for Alzheimer's disease. Alzheimers Dement 2011;7:280-92.

[16] Dubois B, Feldman HH, Jacova C, Cummings JL, Dekosky ST, Barberger-Gateau P, Delacourte A, Frisoni G, Fox NC, Galasko D, et al. Revising the definition of Alzheimer's disease: A new lexicon. Lancet Neurol 2010;9:1118-27.

[17] Khachaturian ZS, Cami J, Andrieu S, Avila J, Boada Rovira M, Breteler M, Froelich L, Gauthier S, Gomez-Isla T, Khachaturian AS, et al. Creating a transatlantic research enterprise for preventing Alzheimer's disease. Alzheimers Dement 2009; 5:361-6.

[18] Thal L, Kuller L, Bowman K, Breitner J, Evans D, Farrer L, Frank R, Khachaturian AS, Khachaturian ZS, Kukull W, et al. The Nevada Vital Aging Initiative: A private-public partnership to study early predictors of dementia. Alzheimers Dement 2007;3:62-7.

[19] Buyse M, Sargent DJ, Grothey A, Matheson A, de Gramont A. Biomarkers and surrogate end points-the challenge of statistical validation. Nat Rev Clin Oncol 2010;7:309-17.

[20] Welch HG, Black WC. Overdiagnosis in cancer. J Natl Cancer Inst 2010;102:605-13.

[21] Greene JA. Prescribing by numbers: Drugs and the definition of disease. Baltimore: Johns Hopkins University Press; 2006.

[22] Borson S, Scanlan JM, Lessig M, DeMers S. Comorbidity in aging and dementia: Scales differ, and the difference matters. Am J Geriatr Psychiatry 2010;18:999-1006.

[23] Borson S. Cognition, aging, and disabilities: Conceptual issues. Phys Med Rehabil Clin N Am 2010;21:375-82. 